Jpn. J. Infect. Dis., 73, 272-277, 2020

Original Article

\title{
Comparison of Loop-Mediated Isothermal Amplification, Microscopy, Culture, and PCR for Diagnosis of Pulmonary Tuberculosis
}

\author{
Benjawan Phetsuksiri ${ }^{1 *}$, Janisara Rudeeaneksin ${ }^{1}$, Sopa Srisungngam ${ }^{1}$, Supranee Bunchoo ${ }^{1}$,Wiphat Klayut ${ }^{1}$, \\ Chie Nakajima ${ }^{2,3}$, Shigeyugi Hamada ${ }^{4,5}$, and Yasuhiko Suzuki ${ }^{2,3}$
}

\begin{abstract}
${ }^{I}$ National Institute of Health, Department of Medical Sciences, Ministry of Public Health, Nonthaburi, Thailand; ${ }^{2}$ Division of Bioresources, Hokkaido University Research Center for Zoonosis Control, Sapporo; ${ }^{3}$ Global Station for Zoonosis Control, Global Institution for Collaborative Research and Education (GI-CoRE), Hokkaido University, Sapporo, Japan; ${ }^{4}$ Section of Bacterial Infections, Research Collaboration Center for Emerging and Re-emerging Infectious Diseases, Nonthaburi, Thailand; and ${ }^{5}$ Research Institute for Microbial Diseases, Osaka University, Osaka, Japan
\end{abstract}

\begin{abstract}
SUMMARY: The diagnosis of tuberculosis (TB) in endemic countries is challenging due to high caseloads and limited resources. A simple and cost-effective diagnostic test for the rapid detection of Mycobacterium tuberculosis (M. tuberculosis) in clinical specimens is crucially needed. We evaluated the performance of an in-house assay based on loop-mediated isothermal amplification (LAMP) targeting the M. tuberculosis 16S ribosomal RNA (rRNA) gene for the diagnosis of TB in Thailand. A total of 252 sputum samples from suspected cases of pulmonary TB were analyzed. The sensitivity of LAMP was 99.04\% (103/104; 95\% confidence interval [CI]: 94.76-9.98\%) and 72.73\% (16/22; 95\% CI: 49.78-89.27\%) for smear-positive and smear-negative samples with TB-culture positivity, respectively. LAMP detected $20.69 \%(24 / 116)$ of TB culture negative samples but all those were positive by conventional polymerase chain reaction (PCR). The sensitivity of LAMP was higher than that of sputum microscopy while the performance of LAMP was similar to PCR. None of the samples positive for non-tuberculous mycobacteria by culture and PCR were positive by LAMP. Compared to TB culture, the positive predictive value (PPV), negative predictive value (NPV), and kappa coefficient of LAMP were $83.22 \%, 88.33 \%$, and 0.75 respectively. Based on the diagnostic performance, we propose that LAMP would be suitable as a potential diagnostic test for rapid TB diagnosis in resourcelimited laboratory settings.
\end{abstract}

\section{INTRODUCTION}

Tuberculosis (TB), caused by the Mycobacterium tuberculosis complex (MTC), is a global health problem. According to the World Health Organization (WHO), an estimated 10 million cases of TB and approximately 1.3 million deaths from the disease were reported in 2017 globally, mostly in the developing countries. Thailand is on the list of $30 \mathrm{~TB}$ high-burden countries and among the top 14 countries with TB, multidrug-resistant (MDR)-TB, and TB-HIV. TB incidence remains high with nearly 108,000 new cases diagnosed and an estimated 156 per 100,000 population including 44,088 new pulmonary bacteriologically confirmed and relapse cases. TB-related mortality including co-infection of HIV and TB is approximately 12,200 in 2017 (1).

Received August 30, 2019. Accepted Februay 3, 2020.

J-STAGE Advance Publication February 28, 2020.

DOI: 10.7883/yoken.JJID.2019.335

*Corresponding author: Mailing address: National Institute of Health, Department of Medical Sciences, Ministry of Public Health, Nonthaburi, Thailand. Tel: 66-2-5801567, Fax: 66-2 9659700, E-mail: benjapsk@health.moph.go.th
Early and accurate diagnosis of TB followed by effective and complete therapy is essential in TB control. The diagnosis of pulmonary TB usually involves clinical evaluation, chest X-ray, and bacteriological culture test. Sputum microscopy is an easy method for screening of TB. However, this method is neither sensitive nor specific for MTC and requires well-trained staff (2). Bacteriological culture is considered the gold standard for TB diagnosis with high sensitivity and specificity, but it is time-consuming, labor-intensive, and requires complex facilities. Therefore, rapid, easyto-use, and accurate TB diagnostic tests are needed for early diagnosis and treatment and to limit further spread of the disease.

Molecular assays based on nucleic acid amplification techniques (NAATs) such as the polymerase chain reaction (PCR) are effective for rapid diagnosis of TB. The Xpert MTB/RIF assay that simultaneously detects both MTC and resistance to rifampin is now more accessible following the WHO policy guidance (3). However, majority of the NAATs currently available are expensive and require complicated devices which limits their use. PCR, which has been applied for the diagnosis of various infectious diseases has certain limitations, including the requirement of thermocyclers, equipment 
for result analysis, and experienced staff. These factors hinder its applicability in resource-limited laboratory settings.

Over the past decade, loop-mediated isothermal amplification (LAMP) assay, a novel NAAT, was developed as a sensitive and cost-effective diagnostic tool (4). Several LAMP assays targeting different MTC genes, including a commercially available LAMP, have been evaluated for the rapid diagnosis of TB and show promise (5-6). LAMP amplifies DNA with high efficiency under isothermal conditions with the high specificity and sensitivity and is easily detected by visual inspection $(4,7)$. Using LAMP for TB diagnosis is feasible and it is necessary to assess its diagnostic performance in high-TB burden settings. In previous studies, we reported the efficacy of the in-house LAMP assay targeting 16S rRNA gene in the identification of MTC in culture isolates (8-10). This study aimed to assess the potential of the in-house LAMP for diagnosis of TB in suspected pulmonary TB patients. The diagnostic performance of LAMP was evaluated to detect MTC in sputum specimens in comparison to smear microscopy as well as Mycobacterial Growth Indicator Tube (MGIT) culture and conventional PCR.

\section{MATERIALS AND METHODS}

Clinical specimens and sample processing: The diagnostic evaluation was conducted at the National Institute of Health, Thailand. The study was performed conforming to the Ethics Committee of Ministry of Public Health, Thailand. For evaluation, smear microscopy, culture confirmation, PCR, and LAMP were performed on sputum specimens obtained from 252 suspected pulmonary TB patients. Presumptive TB cases were identified through clinical and routine radiological TB examination in accordance with the WHO and national TB guidelines. Briefly, patients with productive cough for two or more weeks, and accompanied by one or more symptoms of weight loss, loss of appetite, night sweats, chest pain, fever, shortness of breath, or fatigue and malaise were considered to be presumptive TB cases.

Laboratory tests included conventional microscopy, culture, PCR, and LAMP. Sputum microscopy and TB culture were conducted as a part of routine procedures. Sputum specimens were processed for decontamination using N-acetyl-L-cysteine (NALC)-sodium hydroxide $(\mathrm{NaOH})$ method according to WHO guideline (11). Briefly, an equal volume of NALC- $2 \% \mathrm{NaOH}$ solution was added to the sputum sample and mixed by vortex. Following 15 min incubation at room temperature, decontaminated specimens were concentrated by centrifugation at 3,000 $\times \mathrm{g}$ for $15 \mathrm{~min}$. The supernatant was decanted and the sediment was washed, mixed, and finally re-suspended in $2 \mathrm{ml}$ of phosphate buffer saline. The culture was performed using the MGIT 960 system (Becton Dickinson, Sparks, MD, USA). Positive cultures were confirmed for acid-fast bacilli using ZielhNeelsen $(\mathrm{ZN})$ staining and then underwent identification for MTC using SD Bioline TB Ag MPT64 Rapid kit, an immunochromatographic assay for MPT 64 antigen detection (Standard Diagnostics, Seoul, Korea).

The extraction of DNA from the decontaminated specimens was performed using FlexiGene DNA Kit (Qiagen, CA) with modifications. Briefly, the cell sediment was collected from decontaminated suspension by centrifugation at 13,000 rpm for $5 \mathrm{~min}$. Fifty microliters of FG2 and $0.5 \mu 1$ of proteinase $\mathrm{K}$ were added. The mixture was incubated at $65^{\circ} \mathrm{C}$ for 10 min and then at $95^{\circ} \mathrm{C}$ for another $10 \mathrm{~min}$. To precipitate DNA, $50 \mu 1$ of isopropanol was added and followed by centrifugation at $13,000 \mathrm{rpm}$ for $10 \mathrm{~min}$. After the supernatant was discarded, $50 \mu 1$ of $70 \%$ alcohol was added and followed by centrifugation. The supernatant was discarded and the tube was turned upside down to evaporate any remaining fluid. Finally, $30 \mu 1$ of sterile distilled water were added to dissolve the sediment and the resulting DNA suspension was stored at $-20^{\circ} \mathrm{C}$ for further analysis.

LAMP reaction: The in-house LAMP assay combining a manual DNA extraction, isothermal amplification, and a visual readout was carried out. A set of six LAMP primers (that were assessed for MTC specificity as described in our previous studies) complementary to the nucleotide sequence of the $16 \mathrm{~S}$ rRNA gene was used $(8,12)$. LAMP assay was set up in a final volume of $20 \mu \mathrm{l}$ per reaction. The reaction mixture contained $30 \mathrm{pmol}$ each of the inner primers FIP and BIP, 5 pmol each of the outer primers F3 and B3, 20 pmol each of the loop primers FLP and BLP, $2 \mu 1$ of 10X LAMP buffer $(200 \mathrm{mM}$ Tris- $\mathrm{HCl}$ [pH 8.8], $100 \mathrm{mM} \mathrm{KCl}, 100 \mathrm{mM}\left(\mathrm{NH}_{4}\right)_{2} \mathrm{SO}_{4}$, and $1 \%$ Triton X-100), $1.4 \mathrm{mM}$ deoxynucleotide triphosphates (dNTPs), $0.8 \mathrm{M}$ betaine, $8 \mathrm{mM} \mathrm{MgSO}$, 8 U Bst DNA polymerase (New England Biolabs, Ipswich, MA, USA), and $1 \mu 1$ fluorescent detection reagent (FDR; Eiken Chemical, Tokyo, Japan) along with $6 \mu$ of the DNA lysate. Amplification was performed at $65^{\circ} \mathrm{C}$ for $60 \mathrm{~min}$ in a water bath. Negative control (distilled water) and positive control (MTC H37Ra DNA [0.1 $\mathrm{ng} / \mu \mathrm{l}]$ ) samples were also tested. LAMP results were visualized by naked eye under visible light. A green color or fluorescence indicated a positive reaction, while orange indicated a negative reaction.

The limit of detection (LOD) of the LAMP assay was assessed by 10 -fold serial dilution of the suspension of MTC. The cell suspension was diluted with water to obtain serial cell dilution series from $9 \times 10^{6}-1$ CFU per reaction. The serial dilutions were subjected to DNA extraction and amplification by LAMP as described previously. The LAMP assay was further performed on clinical specimens. Sputum samples that were routinely collected were processed for mycobacterial culture. The remaining processed cell suspension in a total volume of $400 \mu \mathrm{l}$ was subjected to DNA extraction followed by LAMP analysis as described above.

Conventional PCR: For a molecular diagnostic test system comparison, conventional PCR was performed to detect MTC (9). A $439 \mathrm{bp}$-segment of the $65-\mathrm{kDa}$ heat shock protein specific to mycobacteria and a 341 bp-fragment of the desA3 gene specific to MTC were targeted. PCR reaction was performed in a total volume of master mix containing final concentration of $1 \mathrm{U} \mathrm{Taq}$ DNA polymerase, $50 \mathrm{mM} \mathrm{KCl}, 30 \mathrm{mM}$ Tris- $\mathrm{HCl}, 1.5 \mathrm{mM} \mathrm{MgCl} 2$, and $200 \mathrm{mM}$ each dNTP with 100 pmol of primers and DNA template using the remaining DNA in a volume of $6 \mu$ l equal to that used 


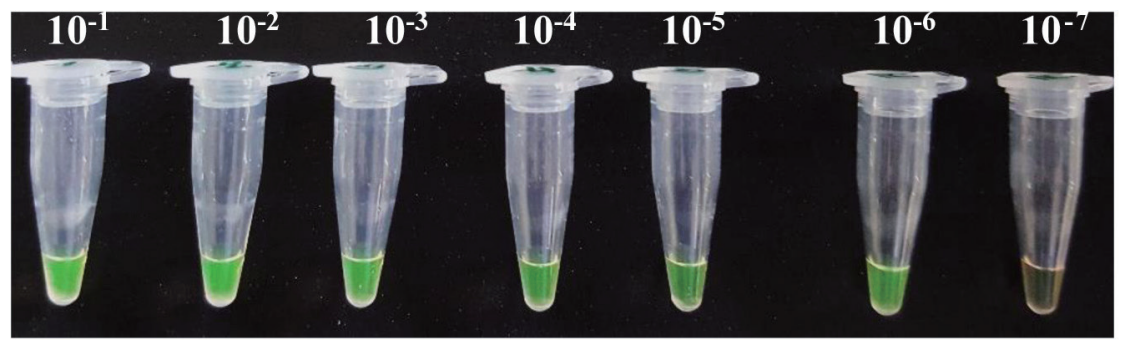

Fig. 1. (Color online) The sensitivity of LAMP. Ten-fold serial dilutions $\left(10^{-1}-10^{-7}\right)$ prepared from cell suspension containing $9 \times 10^{6} \mathrm{CFU}$ was determined by LAMP.

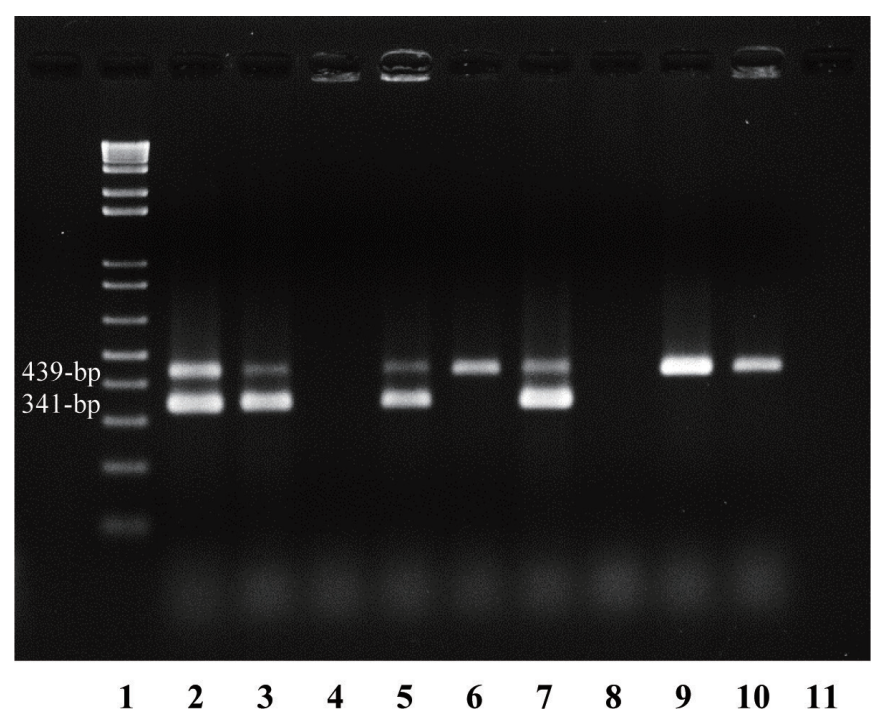

Fig. 2. Detection of mycobacteria in sputum specimens by PCR. The 439 bp-segment is specific for gene encoding $65-\mathrm{kDa}$ heat shock protein in Mycobacterium spp and the 341-bp fragment is specific for desA3 gene in M. tuberculosis. Lanes: 1, 100-bp DNA ladder; 2-3, positive control (M. tuberculosis H37Ra and M. tuberculosis in specimen); 4, negative control; 5-11, sputum specimens; 5, 7, M. tuberculosis; 6, 9-10, Mycobacterium species; 8, 11, negative.

in LAMP. Water was added to the mixture to bring the final volume to $25 \mu 1$. The PCR mixture was amplified in a thermal cycler (Applied Biosystems, Foster City, CA, USA). The cycling protocol consisted of an initial denaturation step at $94^{\circ} \mathrm{C}$ for 5 min, following by 35 cycles of denaturation at $94^{\circ} \mathrm{C}$ for $1 \mathrm{~min}$, annealing at $60^{\circ} \mathrm{C}$ for $1 \mathrm{~min}$ and extension at $72^{\circ} \mathrm{C}$ for $1 \mathrm{~min}$, and a final extension for $10 \mathrm{~min}$. PCR products were analyzed by $1.5 \%$ agarose gel electrophoresis and the gels were stained with ethidium bromide $(0.5 \mu \mathrm{g} / \mathrm{ml})$. A negative control sample was used with each test.

Data analysis: The sensitivity, positive predictive value (PPV), and negative predictive value (NPV), were calculated for LAMP, PCR, and smear microscopy and compared to the culture results. Cohen's kappa ( $\kappa)$ was calculated as a measure of agreement with culture. Concordance between both tests was assessed using the $\kappa$ coefficient. $\kappa$ values below 0.40 indicate weak correlation, values of $0.41-0.60$ indicate good agreement and values above 0.60 indicate strong agreement. Differences were considered significant when $p$ values were less than 0.05 .

\section{RESULTS}

LOD of LAMP reaction: When combined with DNA extraction, LAMP, which is specific for MTC (as reported previously; 8,12 ), could directly detect as few as nine bacilli in LOD analysis (Fig. 1). Based on DNA concentration, the detection limit was equivalent to 45 fg of genomic DNA. The overall LAMP assay protocol allowed MTC detection in a matter of hours without any requirement of expensive or complex instruments. The DNA amplified by LAMP could be directly detected by adding $1 \mu 1$ of FDR to the reaction mixture in the beginning of the preparation of the LAMP mixture. The positive results could be visualized by the naked eye as a color change (Fig. 1).

Microbiological and molecular diagnosis for TB: A total of 252 sputum samples from suspected pulmonary TB patient were analyzed. Of these, $132(52 \%)$ were positive for acid-fast bacilli (AFB) microscopically using $\mathrm{ZN}$ staining and 120 were smear-negative. Upon culturing, 126 samples were positive for MTC and ten samples were positive for non-tuberculous mycobacteria (NTM). In this study, we used multiplex PCR analysis that could differentiate MTC and NTM in a single tube. PCR results that presented a 439-bp DNA band specific 
LAMP for Tuberculosis Diagnosis

Table 1. Positive rate of LAMP in comparison with PCR for detecting M. tuberculosis based on smear and culture results

\begin{tabular}{cccc}
\hline \multirow{2}{*}{ Smear } & Culture & \multicolumn{2}{c}{ Positive rate for MTB $(\%)$} \\
& & LAMP & PCR \\
\hline AFB & MTB & & \\
+ & + & $103 / 104(99.04)$ & $99 / 104(95.19)$ \\
+ & - & $19 / 28(67.86)$ & $28 / 28(100.0)$ \\
- & + & $16 / 22(72.73)$ & $14 / 22(63.64)$ \\
- & - & $5 / 88(5.68)$ & $9 / 88(10.23)$ \\
- & NTM & $0 / 10(0.00)$ & $10 / 10[\mathrm{NTM}]$ \\
Total & & $143 / 252$ & $150 / 252$ \\
\hline
\end{tabular}

Table 2. Diagnostic performance of LAMP test in comparison with AFB staining and PCR based on culture results

\begin{tabular}{|c|c|c|c|c|c|c|c|}
\hline & \multirow[b]{2}{*}{ Results } & \multicolumn{2}{|c|}{ TB-culture $(N=252)$} & \multirow{2}{*}{ Sensitivity $(95 \% \mathrm{CI})$} & \multirow{2}{*}{$\operatorname{PPV}^{1)}(95 \%$ CI $)$} & \multirow{2}{*}{$\mathrm{NPV}^{1)}(95 \% \mathrm{CI})$} & \multirow{2}{*}{ Kappa $^{2)}$} \\
\hline & & Positive & Negative & & & & \\
\hline \multirow{2}{*}{ Smear } & Positive & 104 & 28 & \multirow{2}{*}{$\begin{array}{c}82.54 \\
\text { (74.77 to } 88.72)\end{array}$} & \multirow{2}{*}{$\begin{array}{c}78.79 \\
(72.63 \text { to } 83.87)\end{array}$} & \multirow{2}{*}{$\begin{array}{c}81.67 \\
(75.08 \text { to } 86.82)\end{array}$} & \multirow{2}{*}{0.6} \\
\hline & Negative & 22 & $98^{3)}$ & & & & \\
\hline \multirow{2}{*}{ PCR } & Positive & 113 & 37 & \multirow{2}{*}{$\begin{array}{c}89.68 \\
\text { (83.0 to } 94.39)\end{array}$} & \multirow{2}{*}{$\begin{array}{c}75.33 \\
(69.83 \text { to } 80.12)\end{array}$} & \multirow{2}{*}{$\begin{array}{c}87.25 \\
\text { (80.17 to } 92.06)\end{array}$} & \multirow{2}{*}{0.6} \\
\hline & Negative & 13 & $89^{3)}$ & & & & \\
\hline \multirow{2}{*}{ LAMP } & Positive & 119 & 24 & \multirow{2}{*}{$\begin{array}{c}94.44 \\
(88.89 \text { to } 97.74)\end{array}$} & \multirow{2}{*}{$\begin{array}{c}83.22 \\
(77.53 \text { to } 87.69)\end{array}$} & \multirow{2}{*}{$\begin{array}{c}93.58 \\
\text { (87.59 to } 96.78)\end{array}$} & \multirow{2}{*}{0.75} \\
\hline & Negative & 7 & $102^{3)}$ & & & & \\
\hline
\end{tabular}

${ }^{1)}:$ PPV = positive predictive value, NPV = negative predictive value.

${ }^{2)}$ : Kappa coefficients are shown in number according to interpretive criteria, as follows: 0.21 to 0.4 (fair); 0.41 to 0.60 (moderate); 0.61 to 0.80 (substantial); 0.81 to 1.0 (excellent).

${ }^{3)}$ : Ten isolates were positive for NTM by PCR (shown only 439-bp band).

for Mycobacterium spp. were reported to contain NTM in the samples. Otherwise, PCR amplification that was positive for 439-bp specific mycobacteria and 341-bp specific for MTC was reported as containing MTC in the samples (Fig. 2).

LAMP results for the detection of MTC were compared with the PCR results. Samples positive for MTC by PCR and LAMP were 150 and 143, respectively. All the ten samples containing NTM were NTM-positive by culture and PCR but none of these were positive by LAMP. Therefore, LAMP showed no false-positive results with NTM. The positive rate of LAMP in detecting smear-positive and TB culturepositive pulmonary TB patients was 103 of 104 (99.04\%) while that of PCR was 99 of 104 (95.19\%). On the other hand, the positive rate of LAMP for the diagnosing smear-negative, but TB culture positive cases, was 16 of $22(72.73 \%)$ compared to 14 of $22(63.64 \%)$ for PCR. A total of 116 samples tested negative for MTC and NTM by culture. Of these, 28 were smear-positive and 88 were negative by both microscopy and TB culture. Among TB culture-negative samples, TB-LAMP was positive in $20(20.69 \%)$ samples. However, all those samples were positive for MTC by PCR. The detection results and LAMP positive rate in the different groups of samples are summarized in Table 1.

Based on the culture results, the sensitivity, PPV, $\mathrm{NPV}$, and $\kappa$ coefficient for an agreement were calculated. Compared to culture, regardless to microscopic results, the overall sensitivity, PPV, and NPV of LAMP were 94.44\% (126/143; 95\% confidential interval [CI]: 88.89-97.74\%), 83.22\% (95\% CI: 77.53-87.69\%), and $93.58 \%$ (95\% CI: $87.59-96.78 \%$ ), respectively. The comparable values for smear microscopy were 82.54\% (95\% CI: 74.77-88.72\%), 78.79\% (95\% CI: $72.63-83.87 \%$ ), and $88.2 \%$ (95\% CI: $75.08-86.82 \%)$, respectively. The comparable values for PCR were 89.68\% (95\% CI: 83.00-77-94.36\%), 78.79\% (95\% CI: $69.83-80.12 \%$ ), and $88.2 \%$ (95\% CI: $80.17-92.06 \%$ ), respectively (Table 2). The agreement between LAMP and culture test was good $(\kappa=0.75)$. Table 2 shows the diagnostic performance of LAMP in comparison with microscopy and PCR for pulmonary TB diagnosis.

In subgroup analysis, when the culture was used as a reference standard, we found that the sensitivity of LAMP for smear-positive and negative samples with culture positivity was $99.04 \%(103 / 104 ; 95 \% \mathrm{CI}$ : 94.76-99.98\%) and $72.73 \%$ (16/22; 95\% CI: 49.78$89.27 \%$ ), respectively. Overall, the sensitivity of LAMP in samples positive for MTC by culture was $94.44 \%$ (95\% CI: 88.89-97.74\%; Table 3). 
Table 3. Sensitivity of LAMP in TB-culture positive, smear positive samples vs TBculture positive, smear negative samples

\begin{tabular}{|c|c|c|c|}
\hline & & \multicolumn{2}{|c|}{ Culture (C)-positive } \\
\hline & & AFB (S)-positive & AFB-negative \\
\hline \multirow[t]{2}{*}{ LAMP } & Positive & 103 & 16 \\
\hline & Negative & 1 & 6 \\
\hline \multicolumn{2}{|c|}{ Sensitivity of LAMP for C+, S+ $(95 \% \mathrm{CI})$} & 99.04 (94.76 to 99.5 & \\
\hline \multicolumn{2}{|c|}{ Sensitivity of LAMP for C+, S- $(95 \% \mathrm{CI})$} & $72.73(49.78$ to 89.2 & \\
\hline \multicolumn{2}{|c|}{ Sensitivity of LAMP in overall for C+ } & $94.44(88.89$ to 97.7 & \\
\hline
\end{tabular}

$\mathrm{C}+$, Culture + for MTC; S+, smear + for AFB; S-, smear - for AFB.

\section{DISCUSSION}

LAMP is a manual NAAT that has been recommended by WHO for rapid diagnosis of TB (13). This study was successful in demonstrating the potential of the in-house LAMP for diagnosing pulmonary TB in Thailand, a high-TB burden country. The main finding was the high sensitivity of the inhouse LAMP, which was superior to smear microscopy and similar to PCR. There are several published reports on the diagnostic potential of LAMP assays for the diagnosis of TB. The overall performance varied greatly across studies $(5,14-17)$. LAMP was reported to detect more TB cases than sputum smear microscopy $(2,18)$. Joon et al. reported the sensitivity of various in-house LAMP assays targeting IS6110 gene as varying from $74.88 \%$ to $100 \%$ (19). In addition, Shete et al. reported a sensitivity of $77.7 \%$ and specificity of $98.1 \%$ in meta-analysis of LAMP for pulmonary TB diagnosis (20). Here, the sensitivity of our in-house LAMP for smear-positive/culture-positive and smear-negative/ culture-positive samples were $99.04 \%$ and $72.73 \%$, respectively, which were in the range reported by other studies. The difference in the sensitivity may be due to the efficiency in DNA extraction and LAMP protocols in addition to sample types. Using processed sputum samples may result in an increased concentration of bacilli and hence yield a higher sensitivity compared to unprocessed samples. Our study used concentrated samples, the remainder of samples leftover after the culturing process. Therefore, we could not evaluate the difference in sensitivity between indirect detection using concentrated specimens and direct detection of MTC in sputum samples.

LAMP could detect several cases in smear-negative samples and can therefore be used as a rapid, rule-in test for smear-negative samples. On the other hand, LAMP could detect nearly all smear-positive cases. The undetected cases in the smear-positive samples will require further investigation. Furthermore, it was found that a small proportion of culture-negative samples were positive by LAMP. The discrepant samples that were positive by LAMP but negative by culture were all confirmed to be positive by PCR; thus, we concluded that there were no false-positive results. The suspected causes may involve delayed sample delivery resulting in the reduction of viable cells or no viable cells at the time of culturing. LAMP is probably highly sensitive in the detection of a small number of tubercle bacilli in the patient sputum specimens. In addition, LAMP could detect MTC DNA from non-viable cells which could not be cultivated. Previous studies have also demonstrated a similar problem of high NAAT-positive rates for culture-negative samples $(21,22)$. Therefore, repeating or sample tracking should be performed for all discrepant results.

NAAT has many advantages over conventional microbiological tests including smear microscopy and culture (23). We also compared LAMP to conventional PCR when the culture method was used as a gold standard. The sensitivity of LAMP for TB diagnosis was comparable to that of conventional PCR when the same amount of DNA was used as a template (Table 2). Regarding NTM detection, NTM could be isolated by culture. Meanwhile, NAATs offers an alternative test for rapid differentiation between MTB and NTM in sputum samples. In the present study, none of the samples were positive for NTM by LAMP confirming the high specificity of LAMP for only MTC, as reported previously $(8,12)$. In addition, PPV and NPV of LAMP were more than $80 \%$ and $\kappa$ value was also high. Therefore, based on the overall performance of LAMP and its simplicity, the utility of this assay for detecting MTC in endemic settings is suitable and more feasible for peripheral laboratories.

Acknowledgments This study was supported by the National Institute of Health, Department of Medical Sciences, Ministry of Public Health, Thailand. The work is a part of the program of the Japan-Thailand Research Center Collaboration on Emerging and Re-emerging Infections (RCC-ERI)-Osaka University. The study was also supported by Japan Agency for Medical Research and Development (AMED) and a grant for the Joint Research Program of the Research Center for Zoonosis Control, Hokkaido University from Ministry of Education, Culture, Sports, Science and Technology (MEXT).

Conflict of interest None to declare.

\section{REFERENCES}

1. World Health Organization. Global tuberculosis report 2018. Available at<http://www.who.int/tb/publications/global_report/ en/>. Accessed May 3, 2019.

2. Moon SH, Kim EJ, Tomono J, et al. Detection of Mycobacterium tuberculosis complex in sputum specimens using a loop-mediated isothermal amplification assay in Korea. J Med Microbiol. 2015;64:1335-40. 
3. World Health Organization. Automated real-time nucleic acid amplification technology for rapid and simultaneous detection of tuberculosis and rifampicin resistance: Xpert MTB/RIF assay for the diagnosis of pulmonary and extrapulmonary TB in adults and children. Policy update. Available at $<$ https://www.who.int/tb/ publications/xpert_policyupdate/en/>. Accessed May 3, 2019.

4. Notomi T, Okayama H, Masubuchi H, et al. Loop-mediated isothermal amplification of DNA. Nucleic Acids Res. 2000;28:E63

5. Ou X, Li Q, Xia H, et al. Diagnostic accuracy of the PURELAMP test for pulmonary tuberculosis at the county-level laboratory in China. PLoS One. 2014;9:e94544.

6. Bojang AL, Mendy FS, Tientcheu LD, et al. Comparison of TB-LAMP, GeneXpert MTB/RIF and culture for diagnosis of pulmonary tuberculosis in The Gambia. J Infect. 2016;72:332-7.

7. Notomi T, Mori Y, Tomita N, et al. Loop-mediated isothermal amplification (LAMP): principle, features, and future prospects. J Microbiol. 2015;53:1-5.

8. Rudeeaneksin J, Bunchoo S, Srisungngam S, et al. Rapid identification of Mycobacterium tuberculosis in BACTEC MGIT960 cultures by in-house loop-medicated isothermal amplification. Jpn J Infect Dis. 2012;65:306-11.

9. Phetsuksiri B, Rudeeaneksin J, Srisungngam S, et al. Applicability of in-house loop-mediated isothermal amplification for rapid identification of Mycobacterium tuberculosis complex grown on solid media. Jpn J Infect Dis. 2013;66:249-51.

10. Phetsuksiri B, Rudeeaneksin J, Srisungngam S, et al. Loopmediated isothermal amplification for rapid identification of Mycobacterium tuberculosis in comparison with immunochromatographic SD Bioline MPT64 Rapid® in a high burden setting. Jpn J Infect Dis. 2019;72:112-4.

11. Global Laboratory Initiative. Mycobacteriology Laboratory Manual. Available at $<$ https://www.who.int/tb/laboratory/ mycobacteriology-laboratory-manual.pdf $>$. Accessed May 3, 2019.

12. Pandey BD, Poudel A, Yoda T, et al. Development of an inhouse loop-mediated isothermal amplification (LAMP) assay for detection of Mycobacterium tuberculosis and evaluation in sputum samples of Nepalese patients. J Med Microbiol. 2008;57:439-43.
13. World Health Organization. The use of loop-mediated isothermal amplification (TB-LAMP) for the diagnosis of pulmonary tuberculosis: Policy guidance. Available at $<$ https://www.who.int/ tb/publications/lamp-diagnosis-molecular/en/>. Accessed May 3, 2019.

14. Boehme CC, Nabeta P, Henostroza G, et al. Operational feasibility of using loop-mediated isothermal amplification for diagnosis of pulmonary tuberculosis in microscopy centers of developing countries. J Clin Microbiol. 2007;45:1936-40.

15. Yoshida H, Onohara K, Tazawa T, et al. Comparative study of the efficacy of the COBAS TaqMan and LAMP assay for the rapid diagnosis of tuberculosis. Kekkaku. 2013;88:727-33. Japanese.

16. Kaku T, Minamoto F, D'Meza R, et al. Accuracy of LAMP-TB method for diagnosing tuberculosis in Haiti. Jpn J Infect Dis. 2016;69:488-92.

17. Nagai K, Horita N, Yamamoto M, et al. Diagnostic test accuracy of loop-mediated isothermal amplification assay for Mycobacterium tuberculosis: systematic review and metaanalysis. Sci Rep. 2016;6:39090.

18. Kim CK, Cho EA, Shin DM, et al. Comparative evaluation of the loop-mediated isothermal amplification assay for detecting pulmonary tuberculosis. Ann Lab Med. 2018;38:119-24.

19. Joon D, Nimesh M, Varma-Basil M, et al. Evaluation of improved IS6110 LAMP assay for diagnosis of pulmonary and extra pulmonary tuberculosis. J Microbiol Methods. 2017;139:87-91.

20. Shete PB, Farr K, Strnad L, et al. Diagnostic accuracy of TBLAMP for pulmonary tuberculosis: a systematic review and meta-analysis. BMC Infect Dis. 2019;19:268.

21. Kwak N, Choi SM, Lee J, et al. Diagnostic accuracy and turnaround time of the Xpert MTB/RIF assay in routine clinical practice. PLoS One. 2013;8:e77456.

22. Huh HJ, Jeong BH, Jeon K, et al. Performance evaluation of the Xpert MTB/RIF assay according to its clinical application. BMC Infect Dis. 2014; 14:589.

23. Parsons LM, Somoskövi A, Gutierrez C, et al. Laboratory diagnosis of tuberculosis in resource-poor countries: challenges and opportunities. Clin Microbiol Rev. 2011;24:314-50. 